\title{
Novel Benefits of Remote Ischemic Preconditioning Through VEGF-dependent Protection From Resection-induced Liver Failure in the Mouse
}

Kambakamba, Patryk ; Linecker, Michael ; Schneider, Marcel ; Kron, Philipp ; Limani, Perparim ; Tschuor, Christoph ; Ungethüm, Udo ; Humar, Bostjan ; Clavien, Pierre-Alain

\begin{abstract}
OBJECTIVE To investigate the impact of remote ischemic preconditioning (RIPC) on liver regeneration after major hepatectomy. SUMMARY BACKGROUND DATA RIPC is a strategy applied at remote sites to mitigate ischemic injury. Unlike other preconditioning approaches, RIPC spares target organs as it acts via systemic VEGF elevations. In the liver, however, VEGF is an important driver of regeneration following resection. Therefore, RIPC may have pro-regenerative effects. METHODS RIPC was applied to C57BL/6 mice through intermittent clamping of the femoral vessels prior to standard $68 \%$ hepatectomy or extended $86 \%$-hepatectomy, with the latter causing liver failure and impaired survival. Liver regeneration was assessed through weight gain, proliferative markers (Ki67, pH3, mitoses), cell cycleassociated molecules, and survival. The role of the VEGF-ID1-WNT2 signaling axis was assessed through WIF1 (a WNT antagonist) and recombinant WNT2 injected prior to hepatectomy. RESULTS RIPC did not affect regeneration after $68 \%$-hepatectomy, but improved liver weight gain and hepatocyte mitoses after $86 \%$-hepatectomy. Importantly, RIPC raised survival from $40 \%$ to $80 \%$ after $86 \%$-hepatectomy, indicating the promotion of functional recovery. Mechanistically, the RIPC-induced elevations in VEGF were accompanied by increases in the endothelial transcription factor Id1, its target WNT2, and its hepatocellular effector -catenin. WIF1 injection prior to 86\%-hepatectomy abrogated the RIPC benefits, while recombinant WNT2 had pro-regenerative effects akin to RIPC. CONCLUSION RIPC improves the regenerative capacity of marginal liver remnants in a VEGF-dependent way. If confirmed in patients, RIPC may become the preconditioning strategy of choice in the setting of extended liver resections.
\end{abstract}

DOI: https://doi.org/10.1097/SLA.0000000000002891

Posted at the Zurich Open Repository and Archive, University of Zurich ZORA URL: https://doi.org/10.5167/uzh-165837

Journal Article

Published Version

Originally published at:

Kambakamba, Patryk; Linecker, Michael; Schneider, Marcel; Kron, Philipp; Limani, Perparim; Tschuor, Christoph; Ungethüm, Udo; Humar, Bostjan; Clavien, Pierre-Alain (2018). Novel Benefits of Remote Ischemic Preconditioning Through VEGF-dependent Protection From Resection-induced Liver Failure in the Mouse. Annals of Surgery, 268(5):885-893.

DOI: https://doi.org/10.1097/SLA.0000000000002891 


\title{
Novel Benefits of Remote Ischemic Preconditioning Through VEGF-dependent Protection From Resection-induced Liver Failure in the Mouse
}

\author{
Patryk Kambakamba, MD, Michael Linecker, MD, Marcel Schneider, MD, Philipp Kron, MD, \\ Perparim Limani, MD, PhD, Christoph Tschuor, MD, PhD, Udo Ungethüm, Bostjan Humar, PhD, \\ and Pierre-Alain Clavien, MD, PhD
}

\begin{abstract}
Objective: To investigate the impact of remote ischemic preconditioning (RIPC) on liver regeneration after major hepatectomy.

Summary Background Data: RIPC is a strategy applied at remote sites to mitigate ischemic injury. Unlike other preconditioning approaches, RIPC spares target organs as it acts via systemic VEGF elevations. In the liver, however, VEGF is an important driver of regeneration following resection. Therefore, RIPC may have pro-regenerative effects.

Methods: RIPC was applied to C57BL/6 mice through intermittent clamping of the femoral vessels prior to standard $68 \%$-hepatectomy or extended $86 \%$ hepatectomy, with the latter causing liver failure and impaired survival. Liver regeneration was assessed through weight gain, proliferative markers (Ki67, $\mathrm{pH} 3$, mitoses), cell cycle-associated molecules, and survival. The role of the VEGF-ID1-WNT2 signaling axis was assessed through WIF1 (a WNT antagonist) and recombinant WNT2 injected prior to hepatectomy.

Results: RIPC did not affect regeneration after 68\%-hepatectomy, but improved liver weight gain and hepatocyte mitoses after $86 \%$-hepatectomy. Importantly, RIPC raised survival from $40 \%$ to $80 \%$ after $86 \%$-hepatectomy, indicating the promotion of functional recovery. Mechanistically, the RIPCinduced elevations in VEGF were accompanied by increases in the endothelial transcription factor $I d 1$, its target WNT2, and its hepatocellular effector $\beta$ catenin. WIF1 injection prior to $86 \%$-hepatectomy abrogated the RIPC benefits, while recombinant WNT2 had pro-regenerative effects akin to RIPC. Conclusion: RIPC improves the regenerative capacity of marginal liver remnants in a VEGF-dependent way. If confirmed in patients, RIPC may become the preconditioning strategy of choice in the setting of extended liver resections.
\end{abstract}

Keywords: hepatectomy, liver failure, remote ischemic preconditioning

(Ann Surg 2018;268:885-893)

W hile the liver has the unique capacity to fully regenerate after major resection ${ }^{1-3}$ its regenerative potential is limited by the size of the remnant left after resection. ${ }^{4,5}$ Following excessive

From the Department of Surgery and Transplantation, Swiss HPB and Transplant Center, University Hospital Zurich, Zurich, Switzerland.

$\mathrm{BH}$ and PAC shared senior authorship.

This study was supported by the LGID (Liver and Gastrointestinal Disease) Foundation, Zurich, Switzerland, and through a grant from the Swiss National Science Foundation (S-87002-09-01) and by the Clinical Research Priority Program (CRPP) from the University of Zurich "non-resectable liver tumors from palliation to cure."

The authors report no conflicts of interest.

Supplemental digital content is available for this article. Direct URL citations appear in the printed text and are provided in the HTML and PDF versions of this article on the journal's Web site (www.annalsofsurgery.com).

Reprints: Pierre-Alain Clavien, MD, PhD, Department of Surgery and Transplantation, University Hospital Zurich, Rämistrasse 100, CH-8091 Zurich, Switzerland. E-mail: clavien@ access.uzh.ch.

Copyright (C) 2018 Wolters Kluwer Health, Inc. All rights reserved.

ISSN: 0003-4932/18/26805-0885

DOI: $10.1097 /$ SLA.0000000000002891 hepatectomies, such as necessitated through heavy tumor loads, the marginal remnant cannot recover, leading to liver failure and imminent death. ${ }^{4,6}$ Resection-induced liver failure, better known as small-for-size syndrome (SFSS), is a major factor restricting the surgical management of liver disease. Despite attempts for preventive measures, ${ }^{7}$ the SFSS remains untreatable, representing an open medical need. ${ }^{4,6}$

Since the SFSS is caused by deficient regeneration, ${ }^{3}$ strategies with pro-regenerative potential should be prioritized. Remote ischemic preconditioning (RIPC), a novel approach originally designed to mitigate surgery-associated ischemic injury, ${ }^{8,9}$ may be a candidate. Unlike classic preconditioning approaches, RIPC spares the target organ; in patients, brief periods of ischemia interspersed by reperfusion are induced through transient mechanical obstruction of a limb. Priming ischemia then induces peripheral signals leading to systemic protection. ${ }^{8,9}$

In mice, we have identified VEGF as a key peripheral factor mediating RIPC-induced protection of various organs, including the liver, from ischemic injury. ${ }^{8}$ Although VEGF is long known to participate in liver recovery, more recent evidence indicates VEGF exerts crucial mitogenic effects on hepatocytes after resection. ${ }^{10,11}$ Animated through VEGF, liver sinusoidal endothelial cells (LSECs) induce an ID1-dependent transcription program to produce HGF and WNT2, which then act on hepatocytes to instigate and foster their proliferation. ${ }^{10}$ Given that VEGF is central to the protective effects of RIPC, ${ }^{8}$ the remote strategy may have pro-regenerative effects that might be exploited for the prevention of the SFSS.

To this end, we designed a set of experiments to explore the effects of RIPC on the regeneration of mouse liver after hepatectomy, and to provide a mechanistic basis for a potential regenerative benefit of RIPC. In particular, we assessed the RIPC effects in a mouse model of resection-induced liver failure that features a regenerative deficit, displays minimal ischemic injury, yet recapitulates all key features of the human SFSS. ${ }^{3}$

\section{METHODS}

See Supplement for complete methods.

\section{Experimental Design}

To reveal pro-regenerative effects, RIPC and sham surgery were performed before hepatectomies followed by the assessment of regenerative parameters. Normal, efficient regeneration was induced by $68 \%$-hepatectomy, while extended $86 \%$-hepatectomy - a validated SFSS model - is associated with a regenerative delay causing major metabolic dysfunction (ie, persisting steatosis, hypo-albuminemia, hyperbilirubinemia), liver failure, and high mortality rates. ${ }^{3}$

To explore an association with pro-regenerative VEGF signaling, key components of the paracrine VEGF-ID1-HGF/WNT2CTNNB1 axis were analyzed for RIPC/sham-86\%-hepatectomy. 
To define a role for paracrine WNT2, (i) WNT signaling was inhibited after RIPC by WIF1 (Wnt antagonist) injection, and (ii) RIPC was replaced by recombinant WNT2 injection.

See Supplementary Fig. S1, http://links.lww.com/SLA/B450 for an experimental overview.

\section{Animals}

All animal experiments were in accordance with Swiss federal animal regulations and approved by the cantonal veterinary office of Zurich (Nr. ZH234/14-25781). Male C57B1/6 mice (Envigo, Horst, The Netherlands) aged 10 to 12 weeks were used and kept on a 12-hour day/night cycle with free access to food and water.

\section{Animal Surgery}

Isoflurane anesthesia and buprenorphine analgesia was followed by RIPC. RIPC was applied by clamping the right femoral vascular bundle just proximal to its confluence with the femoral nerve as described ${ }^{8,12}$ and consisted of 3 cycles of 5 minutes of ischemia each followed by 5 minutes of reperfusion (Supplementary Fig. S2, http:// links.lww.com/SLA/B450). Sham RIPC consisted of 30 minutes exposure of the femoral vascular bundle without clamping. Following randomization of animals, standard partial $68 \%$ and extended $86 \%$ hepatectomy were performed as previously described. ${ }^{3}$

\section{Animal Treatment}

Recombinant WIF1 (200 ng/kg, $100 \mu \mathrm{L}$ final vol., R\&D Systems, Minneapolis MI, 135-WF-050) and WNT2 (200 ng/kg, $100 \mu \mathrm{L}$ final vol., Mybiosource, San Diego CA, MBS957358) were injected into the portal vein prior to hepatectomy.

\section{Statistical Analysis}

Five mice/group were included unless stated otherwise. Data are presented as mean \pm SD. Differences were assessed by MannWhitney testing. Survival was assessed by Kaplan-Meier analysis (with $\mathrm{n}=10$ /group, as survival is clinically the most relevant endpoint). Significance was defined as $P<0.05$. GraphPad Prism 6.0 (GraphPad Software Inc, La Jolla, CA) was used.

\section{RESULTS}

\section{RIPC Promotes Liver Weight Gain and Survival in a Model of Resection-induced Liver Failure (SFSS)}

First, we explored the impact of murine hindlimb RIPC on resection-induced liver regeneration. While RIPC had no significant effects following 68\%-hepatectomy (Fig. 1A, Supplementary Fig. S3, http://links.lww.com/SLA/B450), it promoted liver weight gain in our SFSS model (86\%-hepatectomy, Fig. 1B). RIPC further reduced liver injury (assessed through serum AST/ALT, Fig. 1C) after $86 \%$-hepatectomy, although overall injury levels remain low in this model. ${ }^{3}$ No significant improvements were observed for hypoalbuminemia and hyperbilirubinemia (Fig. 1C), however steatosis was markedly ameliorated on histology (Fig. 1D) through RIPC. Next, we assessed 1-week animal survival posthepatectomy, the best measure of functional recovery in the setting of postoperative liver
LWBW $68 \% \mathrm{Hx}$

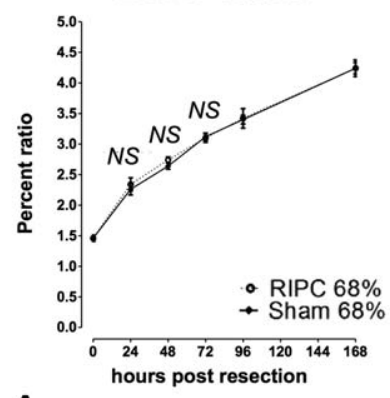

A
LWBW $86 \% \mathrm{Hx}$

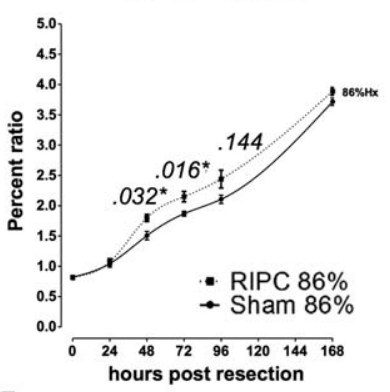

B
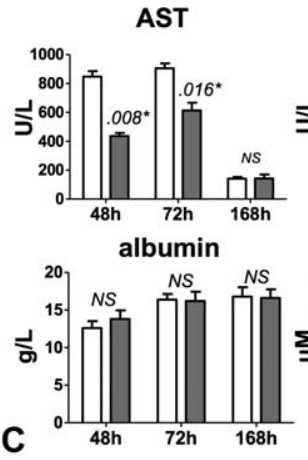

ALT
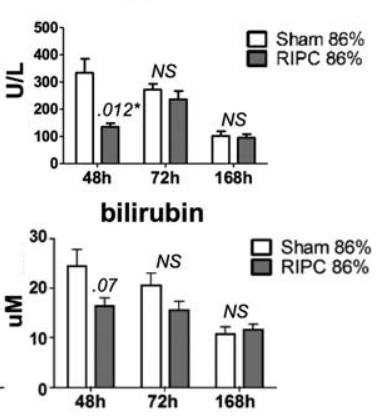
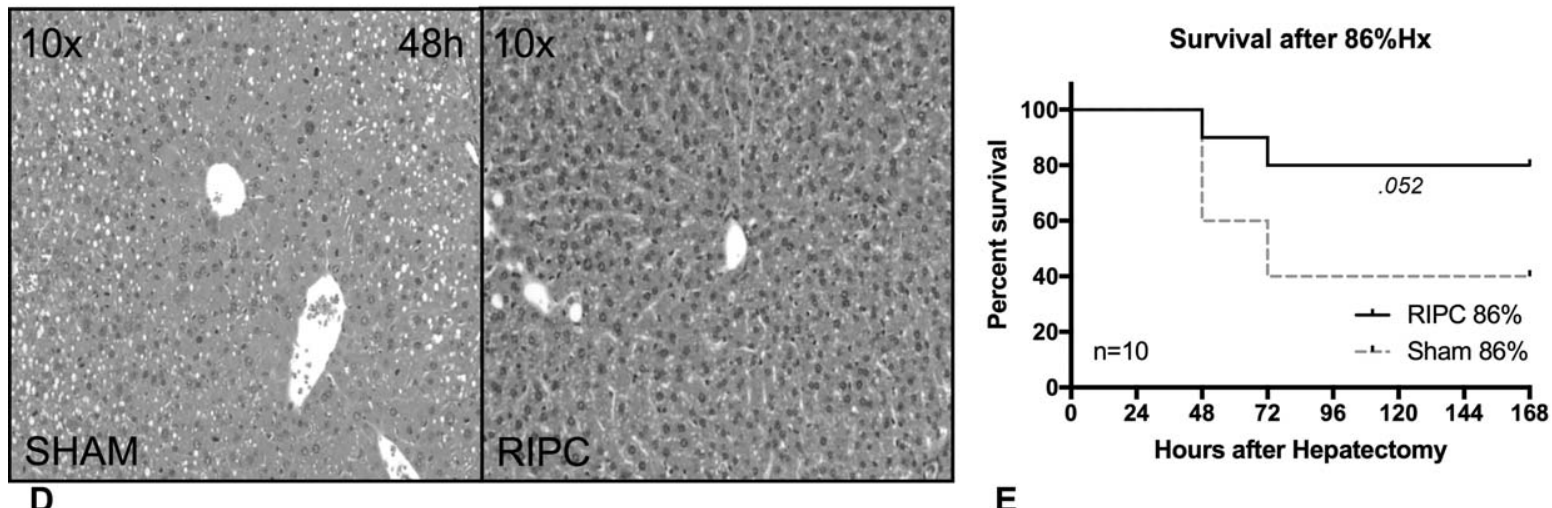

E

FIGURE 1. The impact of RIPC on liver regeneration posthepatectomy. A, Liver weight gain (LW/BW) after RIPC or sham prior to $68 \%$-hepatectomy. B, Liver weight gain (LW/BW) after RIPC or sham prior to $86 \%$-hepatectomy. C, Serum AST, ALT, albumin, and bilirubin levels after RIPC/sham and 86\%-hepatectomy. D, Histology after RIPC/sham and 86\%-hepatectomy. Note the steatotic change in sham-treated liver following 86\%-hepatectomy, a typical SFSS feature. E, Survival after RIPC/sham and $86 \%$-hepatectomy. $\mathrm{N}=5$ /group, Mann-Whitney $U$ testing. For Kaplan-Meier analysis, $\mathrm{n}=10 /$ group was included. 


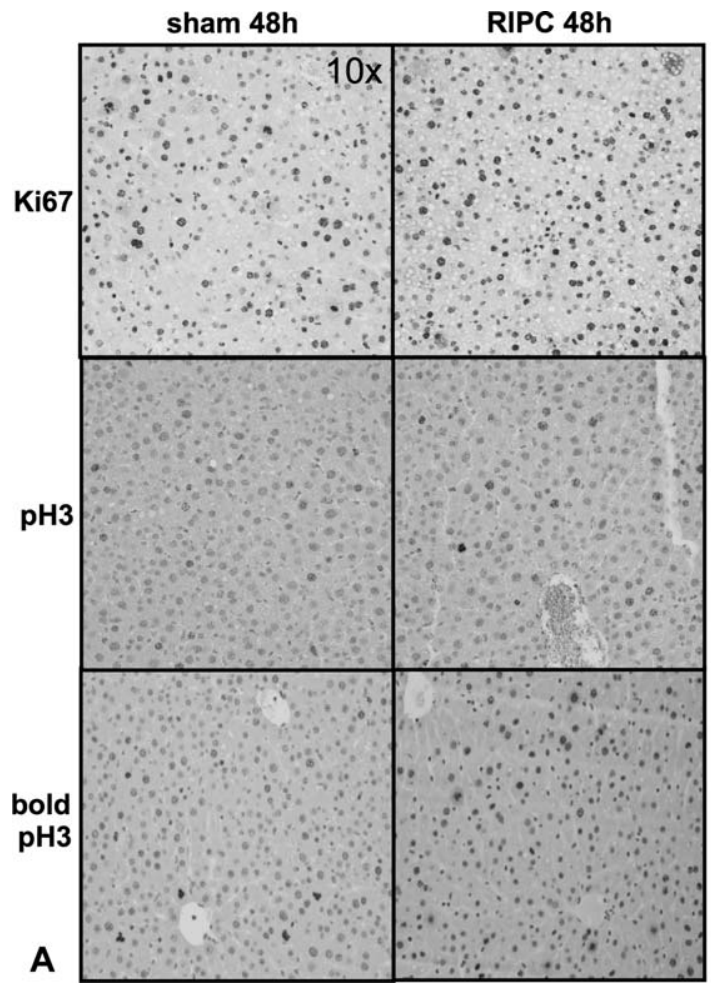

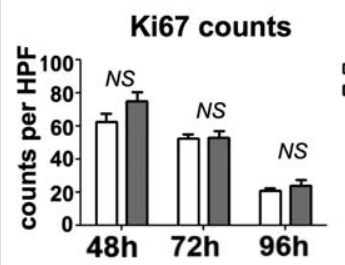
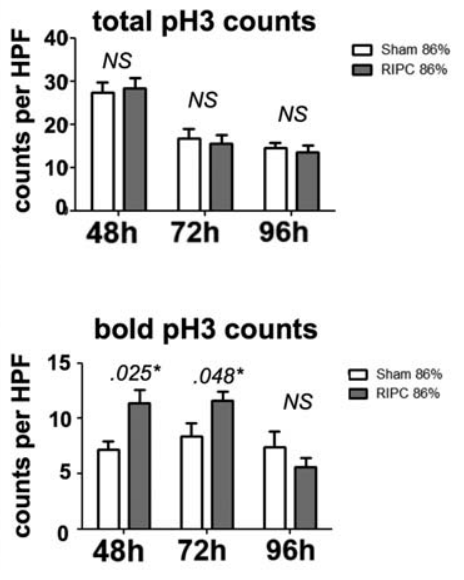
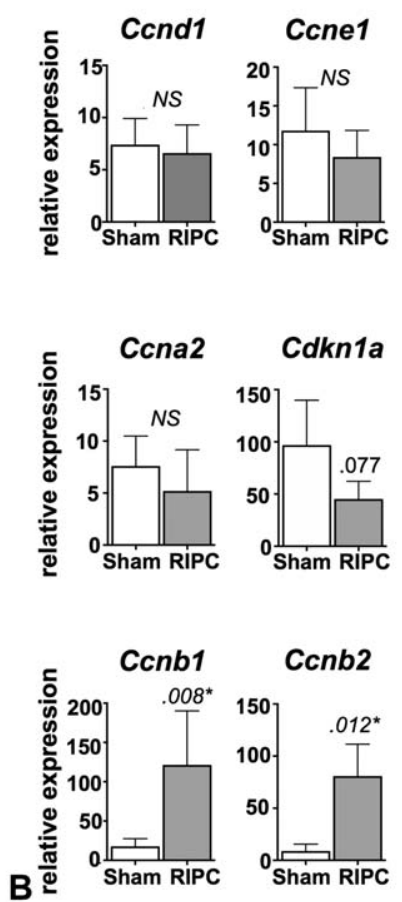

FIGURE 2. Assessment of proliferative parameters after RIPC and 86\%-hepatectomy. A, Immunohistochemistry for hepatic Ki67 (marking all cells in cycle), total $\mathrm{pH} 3$ (marking $\mathrm{G}_{2}$ and $\mathrm{M}$ cells), bold $\mathrm{pH} 3$ (marking $\mathrm{M}$ cells), and corresponding quantifications to the right. Note the significant increases in mitotic cells in liver of RIPC-treated mice. B, Hepatic expression of genes encoding cyclins D1, E1, A2, the cell cycle inhibitor p21, and the mitotic cyclins B1/2 at $48 \mathrm{~h}$ after $86 \%$-hepatectomy in mice pretreated with RIPC or sham. Note the cyclin B upregulation through RIPC. $N=5 /$ group, Mann-Whitney $U$ testing.

failure. ${ }^{3,13,14}$ RIPC doubled survival rates from $40 \%$ in controls to $80 \%$ after $86 \%$-hepatectomy (Fig. 1E). Therefore, RIPC leads to a gain in functional liver mass when applied prior to extended tissue loss.

\section{The Pro-regenerative Effects of RIPC Are Associated With the Promotion of the Hepatocellular Cell Cycle}

SFSS develops due to deficient hepatocellular cell cycle progression. ${ }^{3}$ To determine whether RIPC promotes the cell cycle, we assessed proliferative markers and cell cycle molecules following 86\%-hepatectomy with prior RIPC/sham.

RIPC increased Ki67 counts (marking all hepatocytes in cycle $)^{3}$ without significance, did not affect total phosphohistone 3 (pH3) counts (marking all $\mathrm{G}_{2} / \mathrm{M}$ cells), ${ }^{3}$ but significantly elevated bold $\mathrm{pH} 3$ counts (marking mitotic cells) ${ }^{3}$ particularly at 48 hours posthepatectomy (Fig. 2A), the usual mitotic peak in regenerating liver. ${ }^{3}$ These findings are consistent with an improvement of the SFSS-specific cell cycle defect (ie, normal cell cycle entry but deficient mitosis) ${ }^{3}$ through RIPC. Accordingly, RIPC did not alter the gene expression of early phase cyclins (Ccne1, Ccnd1, Ccna2), but promoted the expression of M-phase cyclins (Ccnb1/b2) at 48 hours post $86 \%$-hepatectomy, along with a marginal reduction in Cdknla encoding the cell cycle inhibitor P21 (Fig. 2B).

We conclude from this 1st set of experiments that RIPC seems to foster regeneration of SFSS grafts by facilitating the progression through mitosis, thereby protecting from liver failure and the associated mortality.

\section{The Pro-regenerative RIPC Effects Are Associated With Paracrine VEGF-ID1-WNT2-CTNNB1 Signaling}

Next, we examined whether the regenerative RIPC benefits are related to VEGF-induced, paracrine ID1-HGF/WNT2-CTNNB1 signaling (Fig. 3A). ${ }^{8,10}$

RIPC alone increased circulating VEGF levels as early as 30 minutes post surgery (Fig. 3A). When combined with $86 \%$ hepatectomy, the RIPC-induced VEGF elevations persisted up to 6 hours after resection (Fig. 3B). In the liver, RIPC led to an increased gene expression of the VEGF-dependent endothelial transcription factor IdI at 24 to 72 hours posthepatectomy, along with elevations of the ID1-targets $H g f$ (significant only at $24 \mathrm{~h}$ ) and Wnt2 (Fig. 3C). At the protein level, hepatic HGF was elevated, albeit without reaching significance $(P=0.092$, Fig. 3C). However, WNT2 protein was significantly upregulated at 48 hours and 72 hours (Fig. 3C). To confirm, liver tissue at 48 hours following RIPC/sham and $86 \%$ hepatectomy was examined by immunohistochemistry, revealing increased WNT2 expression in RIPC- relative to sham-treated liver (Fig. 3D). Finally, nuclear translocation of the WNT effector $\beta$ catenin was promoted in hepatocytes through RIPC at 48 hours and 72 hours after 86\%-hepatectomy (Supplementary Fig. S4, http:// links.lww.com/SLA/B450). Therefore, RIPC promotes peripheral VEGF, the sinusoidal ID1-WNT2 axis, and nuclear $\beta$-catenin 

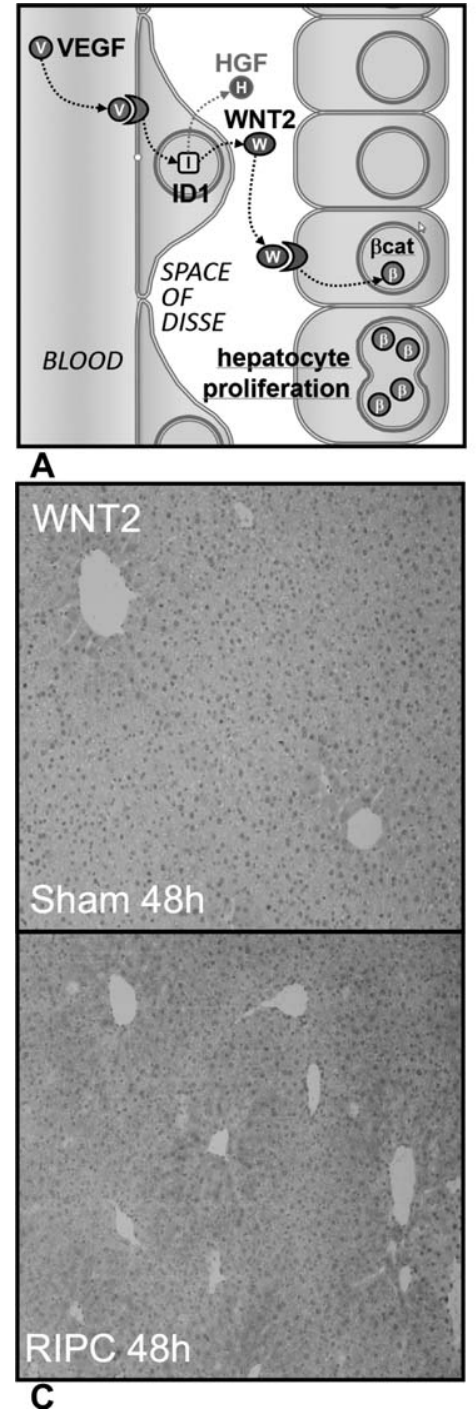

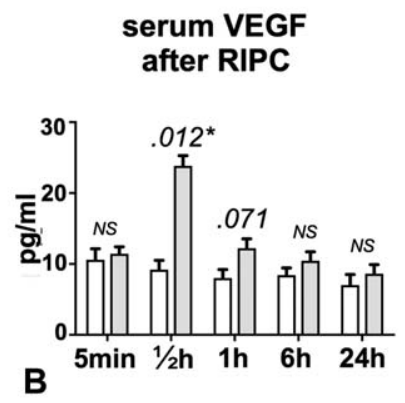

\section{serum VEGF after RIPC and $86 \% \mathrm{Hx}$}

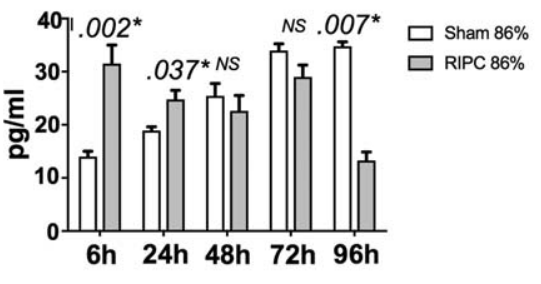

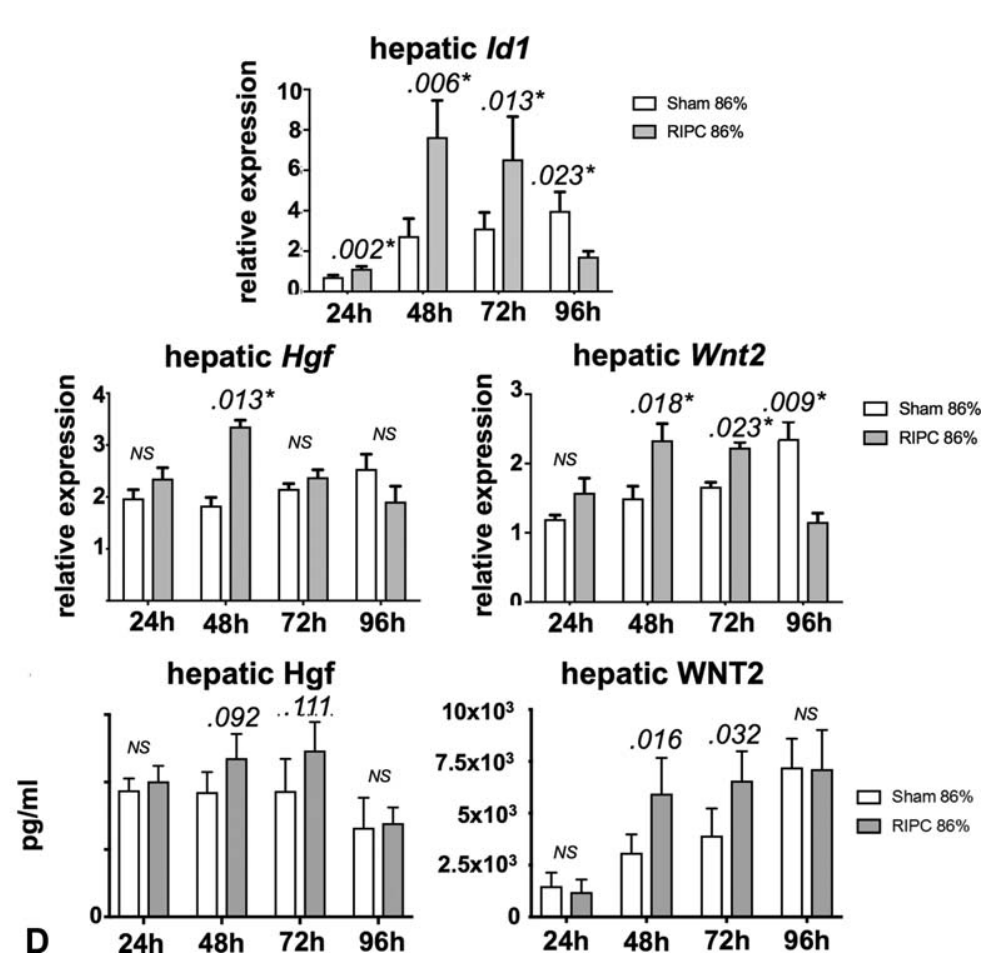

FIGURE 3. Serum VEGF levels and associated hepatic downstream signaling after RIPC and 86\%-hepatectomy. A, The proposed signaling cascade induced by RIPC in hepatectomized mice: a key mechanism underlying the known RIPC effects is the release of circulating VEGF from peripheral endothelial cells. VEGF may then stimulate LSECs to induce via the transcription factor ID1 the production of paracrine HGF and WNT2, with the latter ligand causing nuclear translocation of $\beta$-catenin in hepatocytes to promote their proliferation. B, Serum VEGF levels after RIPC or sham without hepatectomy, and after RIPC/sham and subsequent $86 \%$ hepatectomy. C, Hepatic gene expression levels of Id1, Hgf and Wnt2, and hepatic protein levels of HGF and WNT2 after RIPC/sham and 86\%-hepatectomy. D, Representative immunohistochemical stain for hepatic WNT2 after RIPC/sham and 86\%-hepatectomy. $\mathrm{N}=5$ /group, Mann-Whitney $U$ testing.

translocation in hepatocytes. These findings suggest that the proregenerative effects of RIPC rely on paracrine VEGF-ID1-WNT2CTNNB1 signaling between LSECs and hepatocytes.

\section{The Pro-regenerative and Pro-proliferative RIPC Effects Are Dependent on WNT2}

If RIPC acts through WNT2, WNT2 inhibition should abrogate the RIPC effects, while exogenous WNT2 should mimic RIPC effects.

For WNT2 inhibition, recombinant WIF1 (Wnt inhibitory factor) was injected after RIPC/sham before 86\%-hepatectomy (Fig. 4A). Outcomes were compared to RIPC-86\%-hepatectomy- treated mice injected with saline. WIF1 treatment inhibited liver weight gain at both 48 hours and 72 hours after RIPC-86\%-hepatectomy (Fig. 4B). When recombinant WNT2 was injected before $86 \%$ hepatectomy in the absence of RIPC (Fig. 4A), liver weight was increased akin to RIPC mice relative to saline-injected controls at 72 hours $(P=0.017$, Fig. 4B).

Relative to sham-treated animals, WIF1 treatment of RIPC86\%-hepatectomy mice did not significantly affect $\mathrm{Ki} 67$, total $\mathrm{pH} 3$, and bold $\mathrm{pH} 3$ counts (Fig. 5A), indicating RIPC loses its proliferative effects if WNT2 is inhibited. In contrast, pretreatment of hepatectomized mice with recombinant WNT2 marginally increased Ki67 counts but significantly elevated bold $\mathrm{pH} 3$ counts (Fig. 5A) akin to 

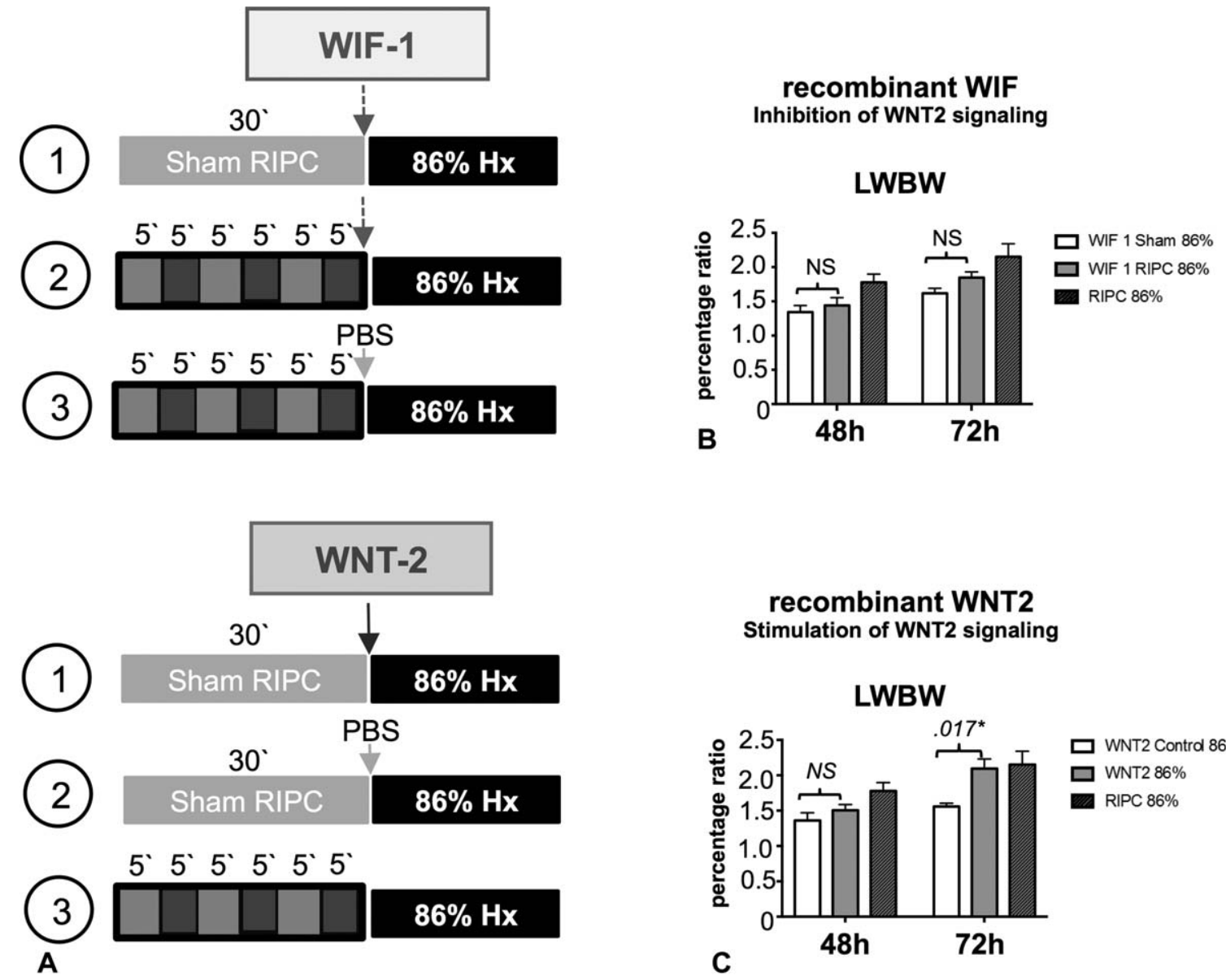

\section{recombinant WNT2 \\ Stimulation of WNT2 signaling}

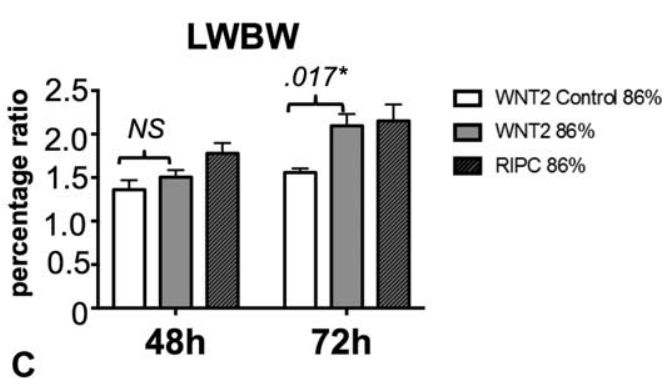

FIGURE 4. The inhibition of RIPC effects through WIF1 and the stimulation of RIPC effects through recombinant WNT2. A, Experimental setup for WIF1 administration after RIPC prior to 86\%-hepatectomy, and WNT2 administration instead of RIPC before hepatectomy. Standard RIPC plus $86 \%$-hepatectomy served as reference. B, Liver weight gain (LW/BW) at $48 \mathrm{~h}$ and $72 \mathrm{~h}$ after sham or RIPC plus WIF1 plus $86 \%$-hepatectomy. C, Liver weight gain at $48 \mathrm{~h}$ and $72 \mathrm{~h}$ after PBS injection (control) plus $86 \%$-hepatectomy, and after WNT2 injection plus 86\%-hepatectomy. $N=5$ /group, Mann-Whitney $U$ testing.

the effects of RIPC (Fig. 2A), confirming that at least some of the pro-proliferative RIPC action is mediated through the ligand. These changes were accompanied by an upregulation of mitotic cyclin genes (Fig. 5B), qualitatively mirroring the RIPC effects on cell cycle molecules (Fig. 2B). Furthermore, WNT2 increased the M-phase promoter Foxm1 and reduced P21 gene expression (Fig. 5B). Again, the impact of WNT2 on B cyclins was less pronounced than that of RIPC, consistent with other contributing molecules or a lesser activity of the recombinant protein. Nonetheless, these findings associate WNT2 upregulation with the promotion of hepatocyte mitosis leading to an improved liver weight gain after $86 \%$-hepatectomy.

Finally, WNT2 treatment, but not RIPC-WIF1 treatment, led to increased nuclear translocation of hepatocellular $\beta$-catenin following 86\%-hepatectomy (Fig. 5C). No significant changes were observed for albumin and bilirubin (data not shown) both of which were not affected by RIPC. The persisting steatosis characteristic of the SFSS was present after WIF1 injection, but was markedly reduced through WNT2 treatment at 48 hours after $86 \%$-hepatectomy (Fig. 5C). Therefore, the pro-regenerative RIPC effects are dependent on WNT2 signaling, while the application of exogenous
WNT2 can mimic most benefits of RIPC in settings of SFSS hepatectomy.

Altogether, our experiments demonstrate that RIPC can foster the regeneration of marginal liver grafts via paracrine VEGF-ID1WNT2 signaling from LSECs, which stimulates hepatocellular mitosis through nuclear $\beta$-catenin translocation.

\section{DISCUSSION}

RIPC is a preconditioning approach unique with regards to its application and its systemic effects. Local ischemia inflicted through RIPC at a limb is sufficient to spread systemic protection from subsequent ischemic stress to various organs. The RIPC effects may extend beyond ischemic protection; in rodent models, RIPC is able to mitigate pancreatitis and hepatic acetaminophen toxicity. ${ }^{8}$ Here, we report a novel benefit of RIPC in mice, which is the promotion of regeneration of small liver remnants after major hepatectomy.

The SFSS is one of the most feared complications after major hepatectomy, leading to deterioration of liver function and even death of the patient. Despite numerous advances in liver surgery, reliable strategies to prevent the SFSS in the clinic are not available thus far. 
WIF inhibition
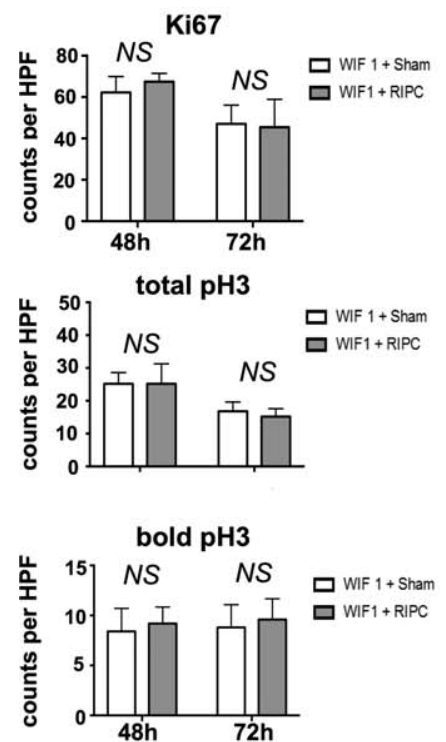

A recombinant WNT2
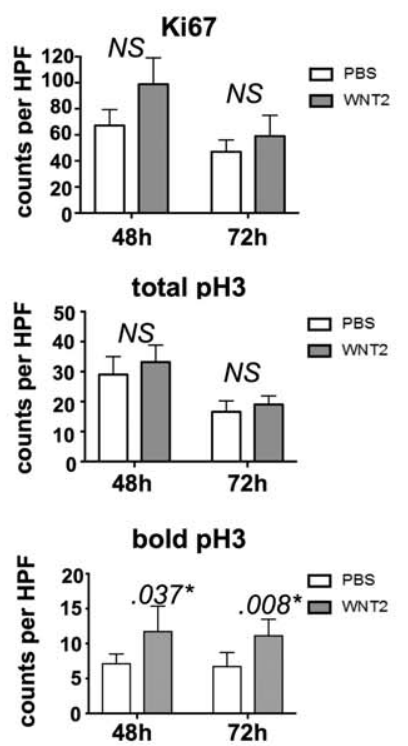

recombinant WNT2
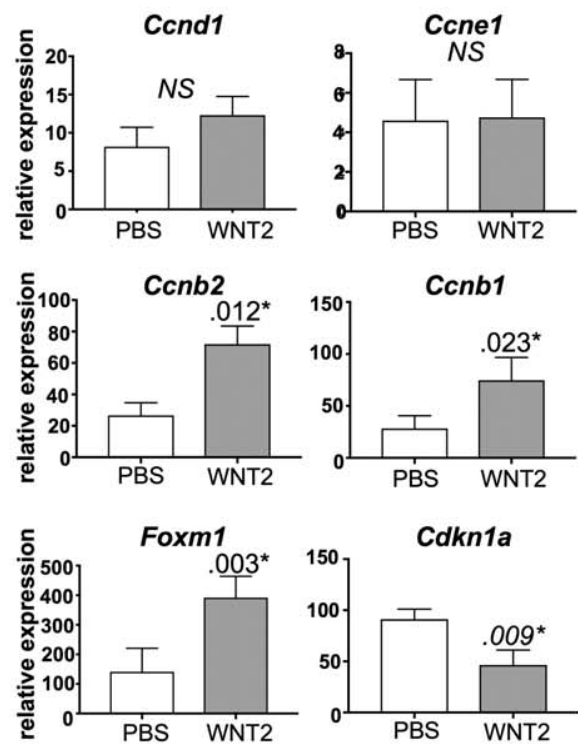

B

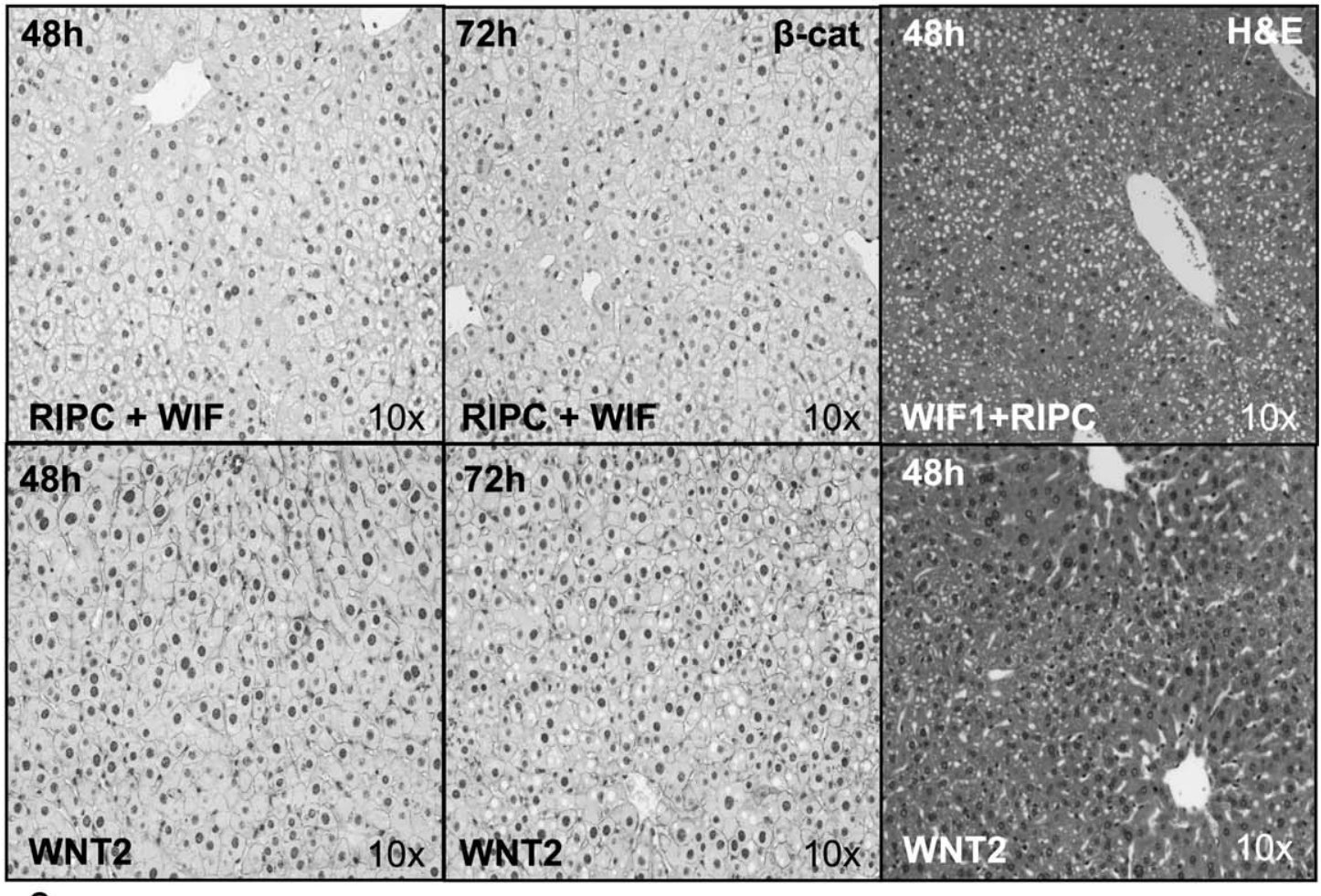

C

FIGURE 5. Assessment of proliferative parameters following RIPC inhibition through WIF1 and following WNT2 administration instead of RIPC. A, Hepatic Ki67, total pH3 and bold pH3 counts after RIPC or sham plus WIF1 and subsequent 86\%-hepatectomy (left panels). Similarly, counts are shown (right panels) for mice subjected to $86 \%$-hepatectomy with concomitant PBS or WNT2 injection. B, Hepatic expression of genes encoding cyclins D1, E1, B1, and B2, the cell cycle promoter FOXM1, and the cell cycle inhibitor P21. Left panels show data for mice subjected to RIPC/sham, WIF1 injection and 86\%-hepatectomy, while right panels show data for mice subjected to PBS/WNT2 injection followed by $86 \%$-hepatectomy. $N=5 / g r o u p$, Mann-Whitney $U$ testing. $C$, Comparison of nuclear $\beta$-catenin expression between liver samples from mice where RIPC effects have been blunted with WIF1 prior to $86 \%$-hepatectomy, and from mice that have been stimulated with WNT2 prior to 86\%-hepatectomy. Note the elevated nuclear staining in WNT2-exposed liver. The histology to the right indicates persisting steatosis in WIF1 - but not WNT2-treated animals. 
In a randomized trial, we have observed that pentoxifylline (PTX, a promoter of the pro-regenerative interleukin-6 pathway) was able to promote the regeneration of small remnants after major hepatectomies. Unfortunately, PTX could not be further considered for clinical application due to unacceptable toxicities associated with this compound. ${ }^{7}$ While liver regeneration is influenced by various patient related (eg, age, underlying liver disease) and surgical (eg, blood loss, ischemia) parameters, we investigated a reproducible mouse model of SFSS that excludes the latter irregularities. ${ }^{3}$ Akin to humans, our model features delayed regeneration, metabolic deficiencies (hypoalbuminemia, hyperbilirubinemia, persisting steatosis), and impaired survival. ${ }^{3}$ Therefore, this model provides an ideal, standardized setting for the study of RIPC effects on marginal liver remnants. In our SFSS model, RIPC was efficient in enhancing hepatocellular mitosis and liver weight gain, while ameliorating steatosis. Although hypoalbuminemia and hyperbilirubinemia remained unaffected, RIPC prior to SFSS hepatectomy enabled doubling of survival, indicating it does promote vital metabolic tasks of marginal liver remnants. Survival remains the most relevant endpoint, particularly as the SFSS is the commonest cause of death due to liver surgery. ${ }^{5}$

VEGF-dependent signaling seems to be central for the RIPC benefits in both ischemia ${ }^{8,12}$ and the SFSS. VEGF-induced angiocrine signals from LSECs have been shown to be pivotal to the regeneration of the parenchyma. ${ }^{10}$ If the VEGF receptor, or its downstream mediator ID1, are deleted in LSECs, hepatocytes display a severe proliferative defect, and liver regeneration is strongly compromised after hepatectomy. This regenerative defect can be corrected by the exogenous provision of HGF and WNT2, the 2 paracrine ligands that are induced by ID1 and act as hepatocyte mitogens. ${ }^{10}$ In analogy, we show that RIPC upregulates the endothelial VEGF-ID1 axis, its target WNT2, and the hepatocellular WNT effector $\beta$-catenin, a key driver of parenchymal proliferation after resection. ${ }^{15}$

Moreover, via modulation of the paracrine mediator WNT2, we demonstrate the importance of this intercellular pathway for RIPC. Altogether, we provide the first evidence that RIPC can exert pro-regenerative effects via paracrine signaling through the $\mathrm{VEGF} \rightarrow \mathrm{LD} 1 \rightarrow \mathrm{WNT} 2 \rightarrow \mathrm{CTNNB} 1$ pathway.

RIPC displayed little effect when applied before standard $68 \%$-hepatectomy. Therefore, RIPC appears not to be able to enhance an already efficient regenerative response as induced in mice by standard hepatectomy. In contrast, extended (86\%) hepatectomy in mouse leads to a P21-dependent arrest of the hepatocellular cycle at the M-phase, ${ }^{3}$ a defect that seems to be specifically targeted by RIPC (ie, elevation of mitotic cyclins, reduction in $\mathrm{P} 21$, promotion of mitotic $\mathrm{pH} 3$ counts). Notably, preliminary findings from our group suggest that WNT2-CTNNB1 signaling is downregulated after $86 \%$ relative to $68 \%$-hepatectomy (Dr Kachaylo, pers.comm.). Therefore, compromised WNT signaling can conceivably explain the efficacy of RIPC specifically in the setting of experimental SFSS.

Besides WNT signaling, RIPC might improve SFSS resections by promoting a healthy LSEC phenotype. Both RIPC and VEGF can maintain open LSEC fenestration, ${ }^{11,12}$ which is needed for a normal regenerative capacity ${ }^{16}$ and reduces exaggerated portal pressure, ${ }^{17}$ a proposed cause of the SFSS. Intriguingly, exogenous VEGF can improve liver regeneration also in a steatotic or cirrhotic background. ${ }^{18-20}$ These findings imply that (i) RIPC may be substituted through VEGF delivery to achieve more targeted benefits, and that (ii) RIPC/VEGF might be beneficial also in a background of liver disease.

RIPC is a recent preconditioning method, and its clinical efficacy is being evaluated. ${ }^{21,22}$ We have initiated a clinical trial assessing the impact of RIPC on postoperative complications associated with major surgical procedures including hepatectomies
(NCT02375269). Meanwhile, experimental data can give hints to estimate the advantages that may be expected from RIPC. Comparing RIPC with intermittent clamping (IC, the gold standard protective strategy) and direct ischemic preconditioning (IPC), we found RIPC superior in the protection of old mouse liver from ischemic injury. ${ }^{12}$ The superior efficacy was associated with enhanced VEGF induction compared with IC, while IPC did not induce detectable VEGF and failed to provide benefit in the aged liver. Notably, IC may promote rat liver regeneration, however only when applied to the lobes to be resected - otherwise deterioration sets in. ${ }^{23}$ In contrast, IPC has been reported to worsen rat regeneration. ${ }^{24}$ Therefore, the available evidence favors RIPC with regards to both its protective and its proregenerative potential, however bound to a background of healthy liver. Whether RIPC will perform also in diseased liver will require additional study. Ultimately, a well-designed trial comparing the different preconditioning approaches in patients undergoing extended resection will be needed to define the clinical utility of RIPC. Importantly, future trials should include all relevant endpoints to assess the efficacy of RIPC against the SFSS. Measures for the prevention of the currently untreatable SFSS represent one of the main challenges in liver surgery, and the ease of RIPC application strongly argues for its clinical feasibility.

In conclusion, we report that RIPC is able to promote the regeneration of marginal liver remnants leading to an improved survival after extended hepatectomy in the mouse. This novel feature is associated with the systemic elevation of peripheral VEGF elicited through RIPC, and relies on the paracrine stimulation of hepatocyte mitosis via VEGF-ID1-WNT2 signaling from LSECs. The proregenerative potential adds to the already described benefits of RIPC, including the non-invasive nature, the sparing of the target organ, the protection beyond ischemic insults, and the efficacy in aged liver. If confirmed in the clinic, RIPC may become the preconditioning method of choice in settings of extended hepatectomies ongoing with an elevated SFSS risk.

\section{ACKNOWLEDGMENTS}

The authors thank Nadia Bain, Eleonora Maurizio, and Pia Fuchs for their excellent technical assistance.

\section{REFERENCES}

1. Michalopoulos GK, DeFrances MC. Liver regeneration. Science. 1997;276: 60-66.

2. Michalopoulos GK. Liver regeneration. J Cell Physiol. 2007;213:286-300.

3. Lehmann K, Tschuor C, Rickenbacher A, et al. Liver failure after extended hepatectomy in mice is mediated by a p21-dependent barrier to liver regeneration. Gastroenterology. 2012;143:1609-1619.

4. Balzan S, Belghiti J, Farges O, et al. The "50-50 criteria" on postoperative day 5: an accurate predictor of liver failure and death after hepatectomy. Ann Surg. 2005;242:824-828.

5. Clavien PA, Petrowsky H, DeOliveira ML, et al. Strategies for safer liver surgery and partial liver transplantation. N Engl J Med. 2007;356:1545-1559.

6. Rahbari NN, Garden OJ, Padbury R, et al. Posthepatectomy liver failure: a definition and grading by the International Study Group of Liver Surgery (ISGLS). Surgery. 2011;149:713-724.

7. Petrowsky H, Breitenstein S, Slankamenac K, et al. Effects of pentoxifylline on liver regeneration: a double-blinded, randomized, controlled trial in 101 patients undergoing major liver resection. Ann Surg. 2010;252:813-822.

8. Oberkofler CE, Limani P, Jang JH, et al. Systemic protection through remote ischemic preconditioning is spread by platelet-dependent signaling in mice. Hepatology. 2014;60:1409-1417.

9. Thielmann M, Kottenberg E, Kleinbongard P, et al. Cardioprotective and prognostic effects of remote ischaemic preconditioning in patients undergoing coronary artery bypass surgery: a single-centre randomised, double-blind, controlled trial. Lancet. 2013;382:597-604.

10. Ding BS, Nolan DJ, Butler JM, et al. Inductive angiocrine signals from sinusoidal endothelium are required for liver regeneration. Nature. 2010; 468:310-315. 
11. Furrer K, Rickenbacher A, Tian Y, et al. Serotonin reverts age-related capillarization and failure of regeneration in the liver through a VEGFdependent pathway. Proc Natl Acad Sci U S A. 2011;108:2945-2950.

12. Limani $P$, Linecker M, Oberkofler CE, et al. Remote ischemic preconditioning: a novel strategy in rescuing older livers from ischemia-reperfusion injury in a rodent model. Ann Surg. 2016;264:797-803.

13. Kachaylo E, Tschuor C, Calo N, et al. PTEN down-regulation promotes betaoxidation to fuel hypertrophic liver growth after hepatectomy in mice. Hepatology. 2017;66:908-921.

14. Tschuor C, Kachaylo E, Limani P, et al. Constitutive androstane receptor (Car)-driven regeneration protects liver from failure following tissue loss. Hepatol. 2016;65:66-74

15. Sodhi D, Micsenyi A, Bowen WC, et al. Morpholino oligonucleotide-triggered beta-catenin knockdown compromises normal liver regeneration. J Hepatol. 2005; $43: 132-141$

16. Ueno S. [An experimental study on the correlation between hepatic hemodynamics and ultrastructural and immunohistochemical changes in sinusoida endothelial cells after major hepatectomy]. Nihon Geka Gakkai Zasshi. 1994;95:248-258.

17. Xu H, Shi BM, Lu XF, et al. Vascular endothelial growth factor attenuates hepatic sinusoidal capillarization in thioacetamide-induced cirrhotic rats. World J Gastroenterol. 2008;14:2349-2357.

18. Bockhorn M, Goralski M, Prokofiev D, et al. VEGF is important for early liver regeneration after partial hepatectomy. J Surg Res. 2007;138:291-299.

19. Gu Y, Sowa JP, Paul A, et al. Vascular endothelial growth factor improves liver regeneration and survival after $90 \%$ hepatectomy in a rat model of dietinduced steatosis. Digestion. 2013;88:235-242.

20. Oe H, Kaido T, Furuyama $\mathrm{H}$, et al. Simultaneous transfer of vascula endothelial growth factor and hepatocyte growth factor genes effectively promotes liver regeneration after hepatectomy in cirrhotic rats. Hepatogastroenterology. 2004;51:1641-1647.

21. Robertson FP, Goswami R, Wright GP, et al. Remote ischaemic preconditioning in orthotopic liver transplantation (RIPCOLT trial): a pilot randomized controlled feasibility study. HPB (Oxford). 2017;19:757-767.

22. Kanoria S, Robertson FP, Mehta NN, et al. Effect of remote ischaemic preconditioning on liver injury in patients undergoing major hepatectomy for colorectal liver metastasis: a pilot randomised controlled feasibility trial World J Surg. 2017;41:1322-1330.

23. Ben Mosbah I, Duval H, Mbatchi SF, et al. Intermittent selective clamping improves rat liver regeneration by attenuating oxidative and endoplasmic reticulum stress. Cell Death Dis. 2014;5:e1107.

24. Eipel C, Glanemann M, Nuessler AK, et al. Ischemic preconditioning impairs liver regeneration in extended reduced-size livers. Ann Surg. 2005;241: $477-484$.

\section{DISCUSSANTS}

\section{Jacques Pirenne (Leuven, Belgium):}

RIPC has been studied more as a strategy against ischemia than to promote regeneration. Distinct mechanisms, among which is the induction of HIF, have been involved in the protection against ischemia. First, is there an overlap between the pro-regenerative and the anti-ischemic effects of RIPC, or are they different?

Have you (or others) used the same RIPC protocol to test resistance to ischemia (liver, kidneys, etc.)?

Second, the SFSS syndrome is caused by insufficient hepatocyte regeneration. It is also caused by an increased portal pressure and endothelial shear stress. In the clinics, maneuvers proven to reduce portal pressure - that is portal shunting - can prevent SFSS. Did RIPC act on portal pressure, too? Is there a possibility that RIPC promoted endothelial protective factors?

Third, the difficulty in translating RIPC from controlled rodent experiments to the clinic is the multifactorial nature of the human setting, and the uncertainty on the length and severity of the ischemia that is required. In this study, you hypothesize that VGEF is the afferent effector. So, why did you not use VGEF directly instead of the relatively crude maneuver of RIPC (arm or limb ischemia)?
Have you tried to infuse VGEF? It would help to prove your hypothesis that VGEF is indeed the primum movens of the proregenerative mechanisms of RIPC. It would also make clinical application easier.

Finally, many livers are discarded for transplantation because of steatosis. I was interested by the reduction of steatosis, which you observed in your treated rodents. What could be the mechanism? Is VEGF involved? It might be worth exploring the effect of RIPC on steatotic livers.

\section{Response From Patryk Kambakamba (Zurich, Switzerland):}

Thank you for evaluating our manuscript and for your questions. I will go through each question point by point.

First, regarding the mechanistic overlap, RIPC induces circulating VEGF elevations, which mediate both its protective and proregenerative effects. Downstream of VEGF, however, the protective RIPC effects are associated with IL-10 and MMP8 elevations in various target organs, which we have previously shown using the same RIPC protocol. ${ }^{1}$ On the other hand, the pro-regenerative RIPC effects depend on VEGF signaling in sinusoidal endothelial cells, which then produce WNT2 to stimulate pro-regenerative WNT signaling in hepatocytes in a paracrine fashion.

Second, elevated portal pressure is often discussed as one of the factors causing the small-for-size syndrome. We did not assess the portal pressure. However, both RIPC ${ }^{2}$ and VEGF $^{3}$ are known to maintain open sinusoidal fenestration, which in turn, is thought to be a prerequisite for normal regeneration ${ }^{4}$ and can lower exaggerated portal pressure. ${ }^{5}$ It is, thus, also likely that RIPC will have beneficial effects on portal pressure.

Third, as for VEGF injections, I believe that the advantage of RIPC is its ease of application, the lack of significant side effects, and the fact that it can be done anywhere. Recombinant VEGF injections will be more costly; however, they might be a better choice for patients, in which RIPC has not achieved sufficient effect (ie, due to insufficient VEGF elevations). Indeed, others have shown that VEGF injections promote liver regeneration. ${ }^{6}$

Finally, given that fat is a primary energy source for the regenerating liver, ${ }^{7}$ we posit that the reductions in regenerationassociated steatosis are a simple consequence of better regeneration that needs more fat. Nevertheless, it would be worthwhile to explore RIPC in steatotic livers, as others have shown improved regeneration of fatty liver following VEGF injection. ${ }^{6}$

\section{Norbert Senninger (Münster, Germany):}

Dr. Kambakamba, thank you very much. I think that your group should be congratulated for bringing new aspects into clinical application. You showed us the proof of principle that the VEGFinduced regeneration of the liver might be helpful. You showed us physiological parameters. I do have a central concern, however, about the effect of the VEGF on the tumor cells. You know about minimal residual disease. Every manipulation of a tumor-bearing liver causes circulating tumor cells, which will be lodged somewhere. While they will eventually be destroyed, they are still present. If you're adding something that makes cells more viable, you're then also perhaps making tumor cells more viable in the process. Did you investigate the effects of VEGF on the tumor cells?

\section{Response From Patryk Kambakamba (Zurich, Switzerland):}

Thank you for this important question. I am not aware of any study associating RIPC with an increased risk of recurrence following liver surgery. I think that RIPC induces only modest and transient 
VEGF elevations, which are sufficient to aid the regenerative response. We would need to investigate whether such elevations will have any impact on tumor cells. However, VEGF is usually associated with hypoxic tumors, and not with circulating, individual tumor cells, such as those released through surgery. Therefore, I believe the associated oncological risks will be acceptable.

\section{Christiane Bruns (Cologne, Germany):}

Thank you very much for the nice presentation. My first question is concerning the methods of the experimental setting. Using the extended partial hepatectomy model, you evaluated a small-for-size syndrome as well as an increase of the remaining liver lobe in relation to the bodyweight of the animals. Could you comment on the bodyweight of the animals that received an extended hepatectomy? I am curious whether the bodyweight of these animals was much less than the control group.

Second, you postulate that the recruited VEGF based on the remote ischemic preconditioning in the limb of the animals is the reason for the effects in the remaining liver lobe after extended partial hepatectomy, which is an endocrine effect. In addition, extended partial hepatectomy causes substantial VEGF upregulation in hepatocytes of the remaining liver lobe. Could you comment on this?

Finally, you measured a substantial activation of WNT signaling. The translocation of cellular WNT into the nucleous triggers a lot of signaling cascades, which promote metastases. I am wondering how you discussed this regarding the transfer of remote ischemic preconditioning into clinical trials, since almost all patients eligible for this concept suffer from primary or secondary liver tumors.

\section{Response From Patryk Kambakamba (Zurich, Switzerland):}

Thank you for these comments.

Concerning your first question, we have to keep in mind that both the RIPC and control groups were subjected to the same extended hepatectomy. Accordingly, remnant-to-bodyweight ratio was similar at hepatectomy for both groups.

Regarding your second question, RIPC elevates both systemic and hepatic VEGF, but I did not show the results of the latter. The main wave of VEGF from hepatocytes, however, occurs at a later stage and serves to promote the regeneration of sinusoids. ${ }^{8}$

Finally, WNT- $\beta$-catenin signaling is well known for its tumorigenic effects. After RIPC, we get a transient and modest nuclear $\beta$ catenin accumulation, which promotes regeneration after an extended hepatectomy, but not after a standard one, pointing to a limited effect of RIPC on $\beta$-catenin activity. In contrast, tumors need chronic $\beta$-catenin over-activation to flourish. Therefore, I believe that the transient $\beta$-catenin activation through RIPC will have little impact on residual tumor cells.

\section{Thomas van Gulik (Amsterdam, The Netherlands):}

I enjoyed your presentation. Congratulations on these very nicely performed experiments. I would also like to elaborate on the role of VEGF a little further. Obviously, when you extrapolate the whole concept of VEGF to the use of liver augmenting techniques to induce liver regeneration, we know that VEGF plays a crucial role.
This is mediated by a mechanism of increased perfusion and concomitant angiogenesis, which is also the reason why tumor growth is stimulated, as a result of neoangiogenesis. So, my question is as follows: why didn't you simply measure the levels of VEGF in your model, and why didn't you look at neoangiogenesis from a histological perspective because this is what happens when you study the effects of VEGF?

\section{Response From Patryk Kambakamba (Zurich, Switzerland):}

Thank you for this question. Certainly, VEGF will have an effect on angiogenesis, but the VEGF-ID1-WNT2 axis has direct mitogenic effects on hepatocytes after resection. ${ }^{9}$ Following an extended hepatectomy, we think that angiogenesis is merely delayed ${ }^{8}$ and not a cause of liver failure. This is why we did not investigate angiogenic activities. However, we assume that the pro-regenerative, hepatocellular effects of RIPC will also normalize angiogenic activities, as successful hepatocyte mitosis is a trigger for the subsequent angiogenic phase after hepatectomy. ${ }^{8}$

\section{References}

1. Oberkofler CE, Limani P, Jang JH, et al. Systemic protection through remote ischemic preconditioning is spread by plateletdependent signaling in mice. Hepatology 2014;60:1409-1417

2. Limani P, Linecker M, Oberkofler CE, et al. Remote Ischemic Preconditioning: A Novel Strategy in Rescuing Older Livers From Ischemia-reperfusion Injury in a Rodent Model. Ann Surg 2016;264:797-803

3. Furrer K, Rickenbacher A, Tian Y, et al. Serotonin reverts agerelated capillarization and failure of regeneration in the liver through a VEGF-dependent pathway. Proc Natl Acad Sci USA 2011;108:2945-2950

4. Ueno S. An experimental study on the correlation between hepatic hemodynamics and ultrastructural and immunohistochemical changes in sinusoidal endothelial cells after major hepatectomy. Nihon Geka Gakkai Zasshi 1994;95:248-258

5. Xu H, Shi BM, Lu XF, et al. Vascular endothelial growth factor attenuates hepatic sinusoidal capillarization in thioacetamideinduced cirrhotic rats. World J Gastroenterol 2008;14:23492357

6. Gu Y, Sowa JP, Paul A, et al. Vascular endothelial growth factor improves liver regeneration and survival after $90 \%$ hepatectomy in a rat model of diet-induced steatosis. Digestion 2013;88:235242

7. Kachaylo E, Tschuor C, Calo N, et al. PTEN down-regulation promotes beta-oxidation to fuel hypertrophic liver growth after hepatectomy in mice. Hepatology 2017;66:908-921

8. Kron P, Linecker M, Limani P, et al. Hypoxia-driven Hif2a coordinates mouse liver regeneration by coupling parenchymal growth to vascular expansion. Hepatology 2016;64:2198-2209

9. Ding BS, Nolan DJ, Butler JM, et al. Inductive angiocrine signals from sinusoidal endothelium are required for liver regeneration. Nature 2010;468:310-315 